\title{
BMJ Open Cohort profile: the China Ageing REespiratory infections Study (CARES), a prospective cohort study in older adults in Eastern China
}

Benjamin J Cowling, ${ }^{1}$ Cuiling Xu ${ }^{1,2,3}$ Fenyang Tang, ${ }^{4}$ Jun Zhang, ${ }^{5}$ Jinjin Shen, ${ }^{6}$ Fiona Havers, ${ }^{7}$ Rachael Wendladt, ${ }^{8}$ Nancy HL Leung, ${ }^{1}$ Carolyn Greene, ${ }^{7}$ A Danielle luliano, ${ }^{7}$ Pat Shifflett, ${ }^{8}$ Ying Song, ${ }^{7}$ Ran Zhang, ${ }^{7}$ Lindsay Kim, ${ }^{7}$ Yuyun Chen, ${ }^{1}$ Daniel KW Chu, ${ }^{1}$ Huachen Zhu, ${ }^{1}$ Yuelong Shu, ${ }^{2,3}$ Hongjie Yu, ${ }^{9,10}$ Mark G Thompson, ${ }^{7}$ on behalf of the CARES investigators

To cite: Cowling BJ, Xu C, Tang F, et al. Cohort profile: the China Ageing REespiratory infections Study (CARES), a prospective cohort study in older adults in Eastern China. BMJ Open 2017;7:e017503. doi:10.1136/ bmjopen-2017-017503

- Prepublication history and additional material for this paper are available online. To view please visit the journal (http:// dx.doi.org/10.1136/bmjopen2017-017503).

Received 5 May 2017 Revised 24 August 2017 Accepted 29 August 2017

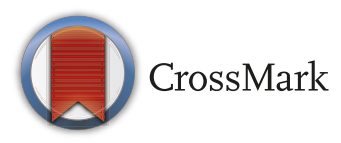

For numbered affiliations see end of article.

Correspondence to Dr Benjamin J Cowling; bcowling@hku.hk

\section{ABSTRACT}

Purpose This study was established to provide direct evidence on the incidence of laboratory-confirmed influenza virus and respiratory syncytial virus (RSV) infections in older adults in two cities in Jiangsu Province, China, and the potential impact of acute respiratory infections on frailty.

Participants The cohort was enrolled in Suzhou and Yancheng, two cities in Jiangsu Province in Eastern China. Between November 2015 and March 2016, we enrolled 1532 adults who were $60-89$ years of age, and collected blood samples along with baseline data on demographics, general health, chronic diseases, functional status and cognitive function through face-to-face interviews using a standardised questionnaire. Participants are being followed weekly throughout the year to identify acute respiratory illnesses. We schedule home visits to ill participants to collect mid-turbinate nasal and oropharyngeal swabs for laboratory testing and detailed symptom information for the acute illness. Regular follow-up including face-to-face interviews and further blood draws will take place every 6-12 months.

Findings to date As of 3 September 2016, we had identified 339 qualifying acute respiratory illness events and 1463 (95\%) participants remained in the study. Laboratory testing is ongoing.

Future plans We plan to conduct laboratory testing to estimate the incidence of influenza virus and RSV infections in older adults. We plan to investigate the impact of these infections on frailty and functional status to determine the association of pre-existing immune status with protection against influenza and RSV infection in unvaccinated older adults, and to assess the exposure to avian influenza viruses in this population.

\section{INTRODUCTION}

Respiratory virus infections are among the leading causes of acute morbidity and mortality globally. ${ }^{1}$ Among the many viruses responsible for acute respiratory infections, influenza virus and respiratory syncytial virus

\section{Strengths and limitations of this study}

- One strength of our cohort is the systematic approach to enrolment with a high enrolment rate, which should permit generalisability of findings to the underlying population, which is older adults residing in rural and urban settings in China.

- Further strengths include the detailed baseline information and stored blood specimens, active surveillance for illnesses and respiratory swabs with high compliance and retention, and electronic capture of study data and activities allowing realtime data quality assurance efforts.

- The weaknesses include the moderate sample size, in the context of relatively low incidence of influenza virus and respiratory syncytial virus infections each year, and the difficulty in preventing response fatigue and dropout.

- A more general limitation of this type of study is that the intensity and impact of influenza epidemics can vary from year to year, and antigenic drift in circulating viruses can hinder analyses of immunity using data pooled across years.

(RSV) infections are thought to be among the most common identifiable causes of acute respiratory hospitalisations and deaths in older adults. ${ }^{2-5}$ Influenza virus and RSV infections occur annually during the winter seasons in the temperate zones of the northern and southern hemispheres and at varying times of the year in tropical and subtropical locations. ${ }^{67}$ The most effective measure to prevent influenza virus infections is vaccination, although influenza vaccine effectiveness is thought to be lower in older adults than in children and younger adults. ${ }^{89}$ An efficacious and safe vaccine for RSV in older adults is anticipated in the near future with candidates in phase I and II clinical trials. ${ }^{10} 11$ 
The WHO recommends that older adults be included as a priority group for influenza vaccination, ${ }^{12}$ and urges member states to attain a coverage of $75 \%$ or higher in older adults. ${ }^{13}$ Despite this, influenza vaccination coverage is low in most locations. ${ }^{14}$ China provides 'Category 1 ' vaccines including routine childhood vaccines, free-of-charge and with high vaccine uptake. However in most parts of China influenza vaccines are provided as 'Category 2' vaccines that are not included in the national immunisation programme, must be paid for out-of-pocket and have low uptake among older adults. ${ }^{15}$ A more comprehensive evidence base on the disease burden of influenza would be valuable for health authorities in China and elsewhere that are considering options to increase influenza vaccination uptake, such as subsidising the cost or providing vaccination free of charge to older adults. Similarly, RSV vaccines for older adults are being developed and data on RSV burden would be essential to guide decisions on future RSV vaccine use.

There are numerous barriers to assessing the spectrum of diseases associated with influenza virus and RSV infections among older adults in low-income and middle-income countries using existing surveillance platforms. Studies that report rates of clinical outcomes such as medically attended influenza and RSV or hospitalisation without laboratory confirmation of influenza and RSV can be difficult to interpret because of coincident circulation of other respiratory pathogens. ${ }^{16}{ }^{17}$ In some countries with both outpatient syndromic influenza-like illness (ILI) surveillance and virological surveillance, the incidence rate of medically attended influenza virus and RSV infections has been estimated by linking syndromic and virological surveillance data for defined catchment populations. ${ }^{18-20}$ However, these studies fail to capture acute respiratory infections that do not meet the ILI criteria (eg, no fever is present) and infections that are associated with mild symptoms not requiring medical attention. Some community-based prospective cohort studies have assessed the incidence rate of clinical illness, as well as influenza virus and RSV infections confirmed by virological or serological testing. ${ }^{21-27}$ Most community-based studies have focused on households with children as the target population; thus, the small samples of older adults enrolled in cohorts to date have resulted in broad uncertainty about estimated incidence rates in this age group. ${ }^{21-24}$

Although most influenza and RSV acute illnesses are self-limiting, some result in severe complications, including hospitalisations and death. ${ }^{28}$ Recent studies have also observed the short-term effects of infections on the risk of cardiovascular complications such as myocardial infarctions and strokes. ${ }^{29}{ }^{30}$ In addition there is some evidence that influenza virus infections may, in some cases, cause a decline in functional status and increase frailty in older adults. ${ }^{31}$ There might be similar longer term consequences of RSV infections. ${ }^{32}{ }^{33}$ However, few longitudinal studies have examined the long-term consequences of influenza virus or RSV infections on frailty, and the contribution of these viral infections to increased frailty in older adults is not well understood. ${ }^{34} 35$

Therefore, we have established a prospective longitudinal cohort study to estimate the incidence of laboratory-confirmed influenza virus and RSV infections in older adults in Jiangsu, China, and the potential impact of influenza virus and RSV infection on frailty and functional status in older adults. In this ongoing study, we assess health of participants including medical conditions and functional status every 6 months, and conduct ongoing active surveillance for acute respiratory illnesses, hospitalisations and death. We collect blood every 6 months, and respiratory specimens during an acute respiratory illness, to permit laboratory confirmation of influenza virus infections during the follow-up period.

There were five reasons for establishing this cohort. The first was to provide direct evidence on the incidence of laboratory-confirmed influenza virus and RSV infections among older adults in two cities in Jiangsu Province, China. This geographical location is part of the Yangtze River Delta in Eastern China, one of the more prosperous areas in China with a population of 140 million persons who have experienced rapid economic development in recent years. Second, we aim to assess the frequency of influenza and RSV disease in a region with multiple epidemic periods; indeed, circulation of influenza in Jiangsu Province varies from year to year, with epidemics in the winter and summer most years (see online supplementary figure 1) ${ }^{36}$ Third, we aim to describe the clinical characteristics of influenza and RSV disease in this population and examine the antibody response to influenza virus infection. The fourth reason is to allow us to investigate the potential impact of influenza virus and RSV infection on frailty and functional status in older adults. The fifth reason is to collect relevant baseline data and establish the feasibility of potential vaccination trials in this location, including trials of twice-annual influenza vaccination or RSV vaccination.

\section{COHORT DESCRIPTION}

This study is an ongoing prospective cohort study in community-dwelling adults 60-89 years of age at enrolment, living in selected urban and semi-urban areas in Jiangsu Province. Additional details of the approaches to enrolment and follow-up of participants and laboratory methods used are provided in online supplementary figure 1, with the questionnaires and data capture forms used (both the used Chinese and the translated English version) provided in annexes (see online supplementary files 1). Briefly, we selected 2 of the 13 major cities in Jiangsu Province, Suzhou and Yancheng, for our study (figure 1 and online supplementary section 1 ). The two cities are located on opposite sides of the Yangtze River (see online supplementary appendix figure 2) and have distinct geographical and demographic characteristics (see online supplementary appendix table $1)$. Suzhou is the second largest city in Jiangsu Province 


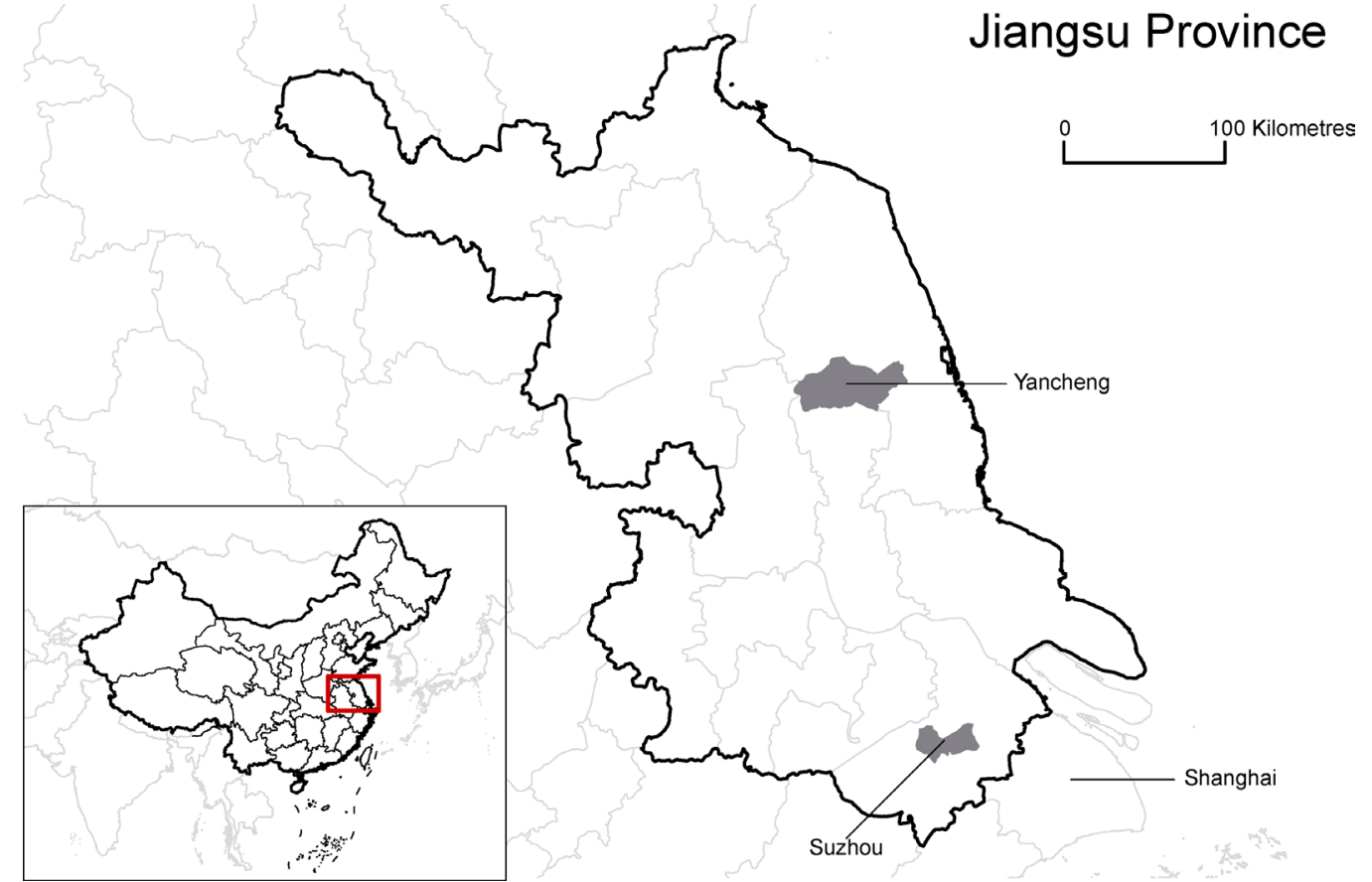

Figure 1 Location of study sites in China.

and has a relatively high gross domestic product of US\$21 634 (¥136 300) per capita, while Yancheng is a rapidly developing city with a moderate gross domestic product of US\$9254 (¥58 299) per capita, which can be compared with the national gross domestic product of US $\$ 7833$ (¥49 351) in China. These two cities were selected based on socioeconomic diversity and the local Centers for Disease Control and Prevention's (CDC) capacity and willingness to collaborate. Within each city, we have included study sites that covered the whole spectrum of urbanisation, including districts that were classified as urban (New District), semiurban (Xiangcheng and Economic Development Zone) and rural (Tinghu) areas, aiming to provide a comprehensive representation of regions with different levels of socioeconomic development in China (see online supplementary appendix table 2). Taken together, the two cities should provide a representative picture of the burden of influenza and RSV in urban-dwelling older adults in Eastern China. We aimed to enrol 1500 older adults with 750 from each city (see online supplementary appendix section 2). A sample size of 1500 participants would permit estimation of an incidence rate of around $2 \%$ each influenza season to within $\pm 0.7 \%$, assuming the mean of the incidence rate follows a normal distribution. We also sought to enrol for the whole cohort 500 participants in each of three age groups: 60-69, 70-79 and 80-89 years old. We elected to oversample the older age groups (of whom there are fewer in the general population) (see online supplementary appendix table 3) so as to provide greater resolution on the incidence and consequences of influenza virus infections among older adults $\geq 80$ years.

We used a combination of recruitment strategies in multiple settings to establish a cohort that was representative of the underlying populations of community-dwelling older adults within each district and for all age strata, and to avoid enrolling a convenience sample that would exclude homebound or chronically ill older adults. All district study sites used a combination of the following three recruitment strategies to identify individuals for screening for eligibility: first, majority was identified by door-to-door visit or telephone contact for older adults in a randomly generated list of registered older adult residents in the district; second, approaching adults attending outpatient clinic appointments for medical care, preventive care or local chronic disease care programme (no more than 20\%); and third, enrolment of older adults via personal referrals by other participants (no more than $10 \%$ ).

Older adults were approached and screened with a standardised form (online supplementary annex 1screening interview) to assess their eligibility for enrolment. Participants had to meet the following inclusion criteria: 60-89 years of age at enrolment, officially reside in the respective city and plan to remain in the study location for at least 2 years, and have a home phone or mobile phone through which the participant can be contacted. Spouses, or two older adults that cohabitate at a single residential address, would both be permitted to join the study, but we would not enrol more than two persons who share the same address to limit potential complications that may rise from clustering or shared risks of exposure and infection. Exclusion criteria included the following: (1) having a bleeding disorder or taking anticoagulant medication (to prevent stroke, pulmonary embolism or deep vein thrombosis) that would preclude blood collection; (2) having an egg allergy (including serious allergic reaction to eggs that required medical attention); (3) 
2280 participants approached

(1103 Suzhou \& 1177 Yancheng)

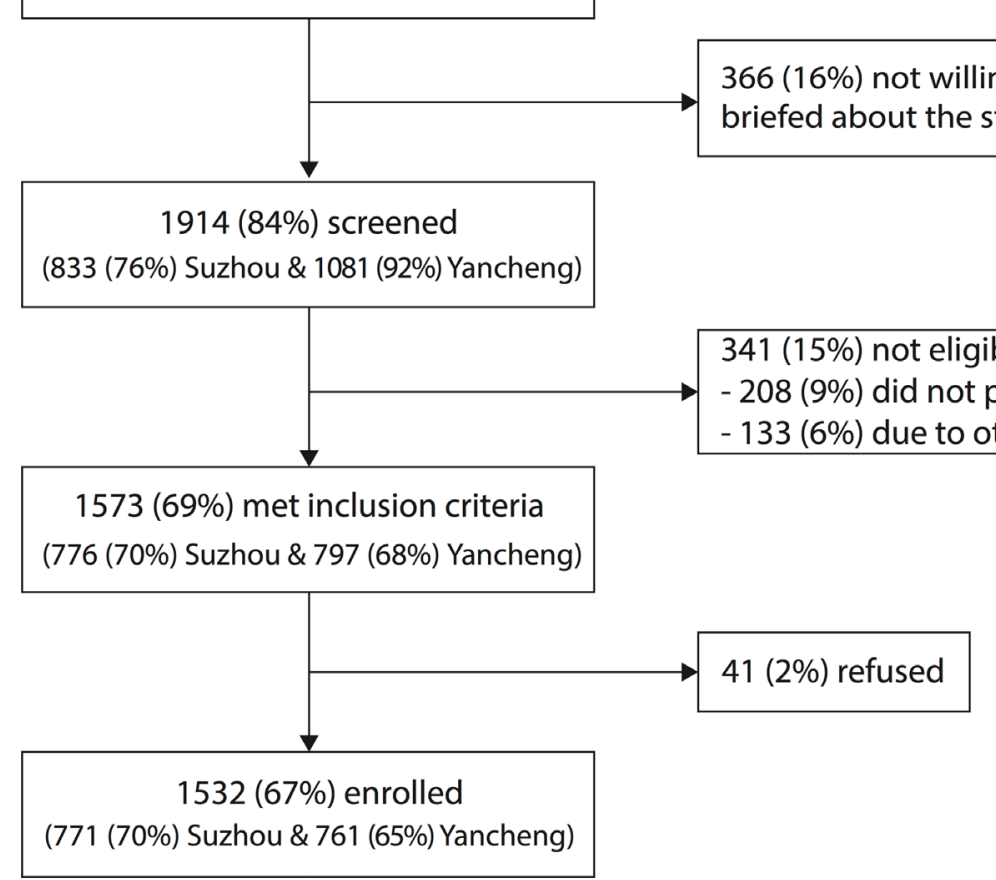

Figure 2 Flow chart of participant enrolment. Among those who were ineligible on screening, 61\% (208/341) were excluded due to failing the cognitive screening, while the remaining $39 \%$ (133/341) were excluded due to 'other reasons', including the following: age $<60$ years or $>89$ years, did not intend to remain in study area, bleeding disorder, use of anticoagulant or could not be contacted by telephone. No individuals were excluded due to having a history of severe reaction to influenza vaccination that required medical attention. Further details on the reasons for refusal or ineligibility are provided in online supplementary appendix tables $7-9$.

having a history of severe reaction to influenza vaccination that required medical attention; (4) showing signs of dementia or significant cognitive impairment; and (5) having a legal guardian/legally authorised representative. The second and third exclusion criteria were specified in order to establish a cohort that would be eligible for future vaccine trials. The fourth and fifth criteria were specified to ensure that participants were only enrolled if they were able to provide informed consent themselves. Otherwise, older adults were enrolled regardless of pre-existing medical conditions.

During the enrolment process, cognitive function was screened using the Mini-Cog tool, ${ }^{37}$ involving threeitem recall and a clock-drawing test (described in detail in online supplementary appendix section 2). Potential participants who were unable to write were permitted to give verbal instructions to a scribe. Older adults were excluded if (1) they were unable to retain and repeat back all three words (after three opportunities); (2) they could not recall any of the words after a several-minute delay and distraction; and (3) they could only recall one or two words after delay and failed the clock-drawing test.

Enrolment began on 18 November 2015; $92 \%$ of enrolment was completed by the end of December 2015, and enrolment was closed on 21 March 2016. We enrolled 1532 older adults in total, with 771 and 761 older adults recruited from Suzhou and Yancheng, respectively. A flow chart of the enrolment process is shown in figure 2. Overall, we screened $84 \%(1914 / 2280)$ of older adults who were approached; $82 \%(1532 / 1914)$ of screened older adults were then enrolled, which included $97 \%$ (1532/1573) of eligible older adults (see online supplementary appendix table 4). Characteristics of the 1532 enrolled participants are shown in table 1, and compared with the 748 older adults who were screened and not enrolled (see online supplementary appendix tables 5 and 6), including 366 older adults who refused to be screened (see online supplementary appendix table 7), 341 who were found not to be eligible (see online supplementary appendix table 8 ), and 41 who were eligible but declined to participate (see online supplementary appendix table 9). The non-enrolled adults had significantly poorer self-rated health and were less likely to have heard of the influenza vaccine. Two hundred and ninety-six out of 771 (38\%) participants in Suzhou and 337 out of 761 (44\%) participants in Yancheng shared the same household with another participant in the cohort.

\section{Baseline demographics, health and functional status}

A schematic of the study activities including specimen collection is illustrated in figure 3. A baseline survey instrument, divided into two parts to streamline the enrolment process, was designed to collect sufficient baseline information to measure potential changes in frailty and 
Table 1 The characteristics of enrolled participants, compared with those approached but not enrolled, in Suzhou and Yancheng

\begin{tabular}{|c|c|c|c|}
\hline & Enrolled & Approached but not enrolled ${ }^{\star}$ & \\
\hline & $(n=1532)$ & $(n=748)$ & \\
\hline & n (\%) & n (\%) & p Value \\
\hline \multicolumn{4}{|l|}{ Sex } \\
\hline Male & $680(44.4 \%)$ & $327(43.7 \%)$ & 0.80 \\
\hline Female & $852(55.6 \%)$ & $421(56.3 \%)$ & \\
\hline \multicolumn{4}{|c|}{ Age group, in years } \\
\hline$<60$ & $0(0)$ & $31(8.1 \%)$ & $<0.01$ \\
\hline $60-69$ & $548(35.8 \%)$ & $110(28.8 \%)$ & \\
\hline $70-79$ & $518(33.8 \%)$ & $125(32.7 \%)$ & \\
\hline $80-89$ & $466(30.4 \%)$ & $110(28.8 \%)$ & \\
\hline$\geq 90$ & $0(0)$ & $6(1.6 \%)$ & \\
\hline \multicolumn{4}{|c|}{ Self-reported health status } \\
\hline Excellent & $27(1.8 \%)$ & $1(0.3 \%)$ & $<0.01$ \\
\hline Very good & $316(20.6 \%)$ & $14(4.4 \%)$ & \\
\hline Good & $581(37.9 \%)$ & $41(13 \%)$ & \\
\hline Fair & $554(36.2 \%)$ & $175(55.6 \%)$ & \\
\hline Poor & $54(3.5 \%)$ & $84(26.7 \%)$ & \\
\hline \multicolumn{4}{|c|}{ Heard of influenza vaccine } \\
\hline Yes & $344(22.5 \%)$ & $16(5.1 \%)$ & $<0.01$ \\
\hline No & $1188(77.5 \%)$ & 299 (94.9\%) & \\
\hline \multicolumn{4}{|c|}{ Self-reported ever received influenza vaccine } \\
\hline Yes & $13(0.8 \%)$ & $2(0.6 \%)$ & $>0.99$ \\
\hline Not & $1519(99.2 \%)$ & 313 (99.4\%) & \\
\hline
\end{tabular}

*Older adults approached but not enrolled were excluded at multiple stages. Therefore, the total numbers in each category do not always add up to 748 , but reflect the numbers of the approached/screened who have responded to the specific question.

†Older adults who have not heard of influenza vaccine were assumed to have never received influenza vaccine.

functional status over time, as well as other relevant demographic and clinical information: at enrolment, a short questionnaire (online supplementary annex 2enrolment interview 1) was administered immediately after written informed consent was obtained; and within 1-3 months following enrolment, a second face-to-face encounter was arranged (from 25 December 2015 to 12 May 2016) to administer a longer structured questionnaire (online supplementary annex 3-enrolment interview 2) to collect detailed baseline information on cognitive function, life history, socioeconomic status (SES), general health, underlying medical conditions, functional status, and participants' knowledge, attitudes and practices relating to influenza vaccination (see online supplementary appendix section 3). Medical conditions recorded included cardiovascular diseases, chronic lung diseases, kidney diseases, liver diseases, cancers, depression or anxiety disorders, diabetes, neurological disorders, osteoarthritis, autoimmune diseases and any other conditions that were reported by the participants. All participants completed standardised questionnaires and the full Standardised Mini-Mental State Examination (SMMSE). ${ }^{38}$ To gather in-depth information, half of the participants were randomly assigned to receive an extended assessment of SES and health, and the other half received an extended assessment of functional status (see online supplementary appendix table 10). The extended SES and health assessment included subjective social status, ${ }^{39} 40$ perceived financial deprivation, ownership of home and appliances, and a visual analogue assessment of current health. ${ }^{41}$ The extended functional status assessment assessed ability to do activities of daily living using questions from the Groningen Activity Restriction Scale, ${ }^{42}$ the Tilburg Frailty Indicator, ${ }^{43} 44$ the Groningen Frailty Indicator, ${ }^{45}$ the Lawton Instrumental Activities of Daily Living Scale ${ }^{46}$ and the Behavioral Risk Factor Surveillance System 2014 questionnaire. ${ }^{478}$ All participants were asked about whether they needed help to do activities (sometimes, often, very often or all the time) or could do them independently; those who received the extended version were also asked whether independent activities were done with difficulty (without any difficulty, with some difficulty or with great difficulty). Scores on these questions, as well as the SMMSE and medical history, will be used to calculate a 
(A)

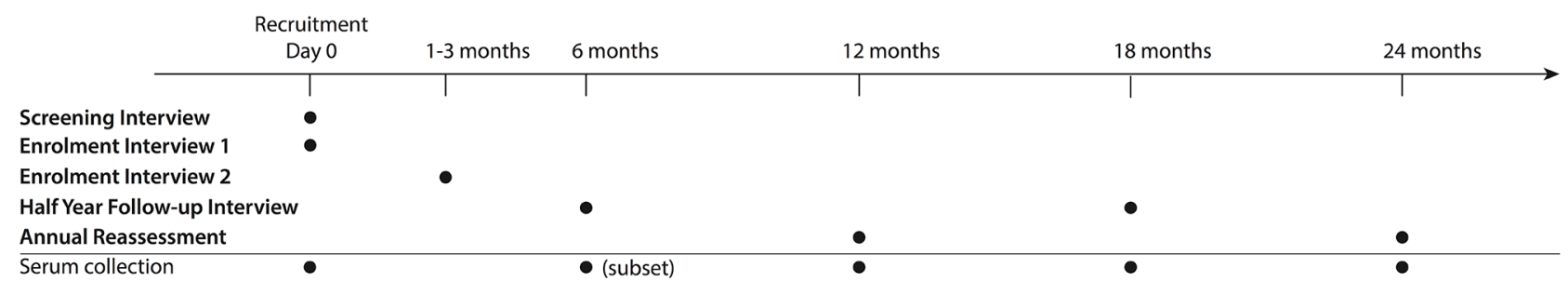

(B)

\begin{tabular}{|c|c|c|c|c|c|c|}
\hline $\begin{array}{l}\text { Illness } \\
\text { onset } \\
\text { Day } 0\end{array}$ & $\begin{array}{l}\text { Reporting } \\
\text { illness }\end{array}$ & $\begin{array}{l}\text { After home } \\
\text { visit }\end{array}$ & 10 days & 13 days & Hospitalisation* & Death* \\
\hline \multirow[t]{2}{*}{7} & $T$ & 1 & $T$ & T & $7^{-1}$ & \\
\hline & $\begin{array}{l}\text { Home visit (within } 7 \text { days): } \\
\text { Administering Acute } \\
\text { Illness Interview } \\
\text { Staff collecting swabs }\end{array}$ & $\begin{array}{l}\text { Participants fill in daily } \\
\text { Symptom Diary }\end{array}$ & $\begin{array}{l}\text { Telephone contact } \\
\text { for illness resolution, } \\
\text { using Illness } \\
\text { Follow-up Interview }\end{array}$ & $\begin{array}{l}\text { If illness not resolved, } \\
\text { continue telephone contact } \\
\text { every } 3 \text { days for up to } \\
4 \text { times or till resolution }\end{array}$ & $\begin{array}{l}\text { Complete } \\
\text { Hospital Case } \\
\text { Report Form }\end{array}$ & $\begin{array}{l}\text { Complete } \\
\text { Death Record }\end{array}$ \\
\hline
\end{tabular}

Figure 3 Timelines of major study activities. (A) Timeline of administration of survey instruments and blood collection in all participants. Older adults were screened for eligibility with the screening interview (online supplementary annex 1), and enrolled participants provided basic baseline information at enrolment with the enrolment interview 1 (online supplementary annex 2) and detailed baseline information within 1-3 months after enrolment with the enrolment interview 2 (online supplementary annex 3). Updated information is captured briefly with the half year follow-up interview (online supplementary annex 4) during mid-year encounters and more extensively with the annual reassessment (online supplementary annex 5) during end-of-year encounters in each study year. Self-reported medical histories collected at baseline and regular reassessments will be validated with those abstracted from medical records using the standardised medical chart review form (online supplementary annex 6) on regular basis (not shown here). Clotted blood for serum is collected from all participants every 6 months from enrolment, except at the sixth month when blood was collected from a subset (about $50 \%$ ) of participants instead. A participant withdrawal form (online supplementary annex 13) will be administered at time of exit (not shown) when he/she seeks to withdraw from the study at any point of the study. (B) Timeline of study activities during an illness episode in participants who report qualifying illness. We actively identify acute respiratory illnesses and hospitalisations throughout the study period. Once a qualifying illness is identified with the symptom screening log (online supplementary annex 8, not shown here), we arrange home visit to collect respiratory specimens (mid-turbinate nasal and oropharyngeal swabs) within 7 days and information on the illness severity with the acute illness interview (online supplementary annex 9) within 10 days from illness onset. Approximately 10 days after illness onset, research staff telephones the participant to ask if the illness has resolved and ask about illness when the participant is most ill with the illness follow-up interview (online supplementary annex 10). Participants who have not recovered at this time will be telephoned every 3 days (up to four times) or until an illness resolution date is identified. A small number of participants fill in a symptom diary (online supplementary annex 11) to describe their illness symptoms on each day during the illness. When we discover a participant is hospitalised due to respiratory causes, we will abstract relevant information from hospital records with the hospital case report (online supplementary annex 7). Hospitalisations in the past month are also actively identified with the monthly hospitalisation surveillance form (online supplementary annex 14) on a monthly basis since April 2017 (not shown here). When we discover a participant has passed away, we will obtain relevant information from family members willing to share this information or abstract from medical records and death certificates with the death record (online supplementary annex 12). The asterisk by hospitalization and deaths indicates that relevant information was only collected from participants that were hospitalized or died, respectively.

Frailty Index. ${ }^{49}$ Table 2 summarises the times at which these and other data are collected.

\section{Regular reassessments of demographics, health and functional status}

During the summer of 2016, a brief questionnaire (online supplementary annex 4-half year follow-up interview) was administered to collect updated information on health and functional status, and a similar questionnaire will be used in the mid-year encounter in the second year (see online supplementary appendix section 4). During each September-December encounter, a longer annual reassessment questionnaire (online supplementary annex 5-annual reassessment) will be administered to collect updated information on demographics and household background, influenza vaccination, general health/chronic diseases and functional status, as well as SMMSE in even-numbered years. We plan to validate the self-reported medical histories collected at baseline and regular reassessments with those abstracted from medical records using a standardised data abstraction tool (online supplementary annex 6-medical chart review and online supplementary appendix section 7).

\section{Active surveillance for illnesses}

Starting 28 December 2015 in Suzhou and 11 January 2016 in Yancheng, we have been conducting active surveillance for respiratory illnesses all year round including once-weekly telephone contact with each participant (see online supplementary appendix section 5), which permits home visits for collection of nasal and throat swabs during an acute illness for testing by real-time reverse 


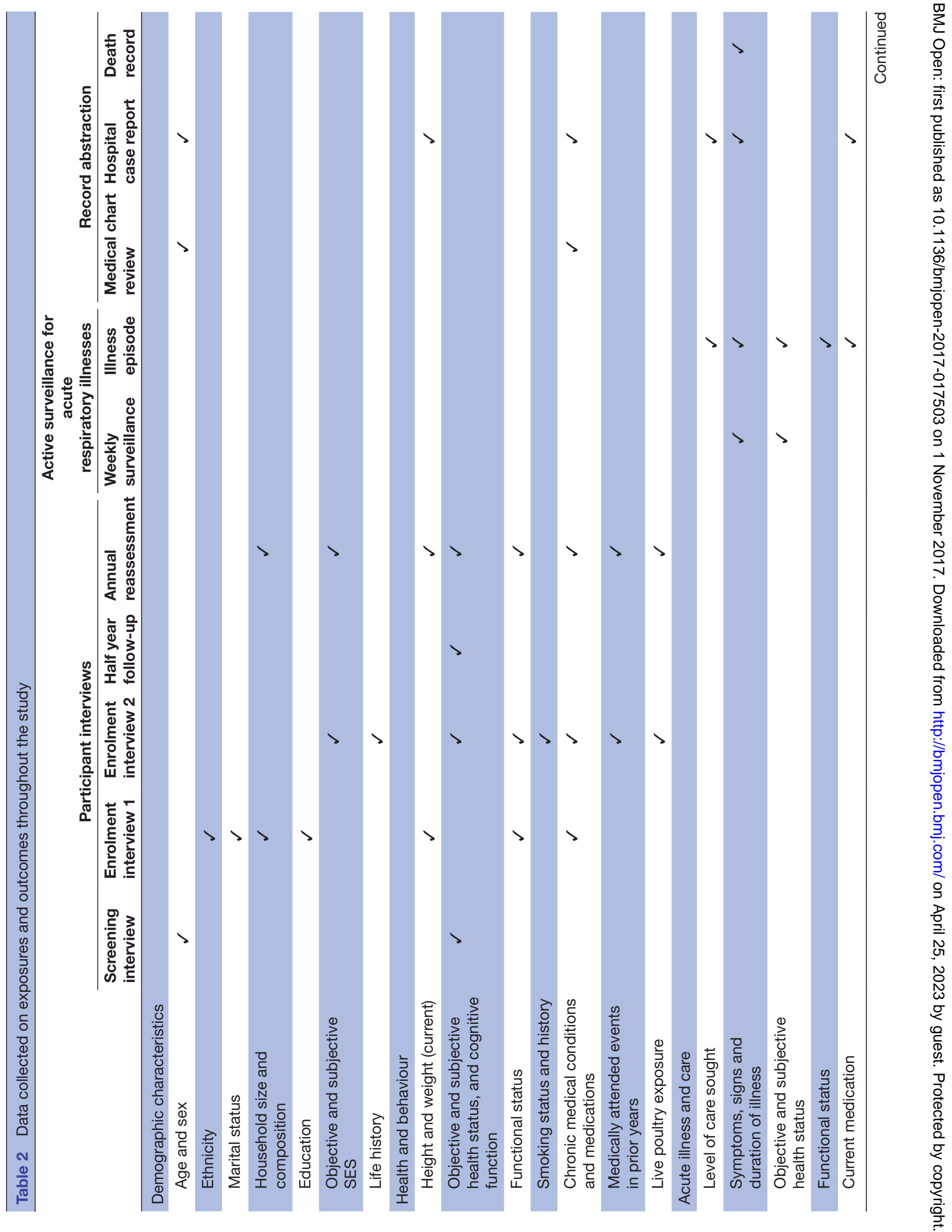


transcription PCR (rRT-PCR) to confirm influenza virus and RSV infections, and completion of illness surveys on symptom severity, cognitive function, subjective health status, medical care and daily activities during the early and late stages of the acute illness (see online supplementary appendix section 6). In Suzhou, during periods of heightened local influenza circulation as determined from a review of local influenza surveillance data, ${ }^{50}$ study staff call participants twice every week. In Yancheng, call frequency remains once-weekly throughout the year. For the small subset of participants without their own telephones, surveillance calls are placed to their caregivers, children or neighbours who are willing to answer for them. When an illness is reported by a participant, research staff complete a brief screening (online supplementary annex 8-symptom screening log) to identify symptoms and the illness onset date. Depending on the number of symptoms identified, the illness onset date and whether the illness has already resolved, different surveillance activities (illness surveys with or without respiratory specimen collection) will be completed (see online supplementary appendix table 12). A qualifying illness is defined as an illness with at least two respiratory symptoms (fever/feverishness, runny nose, worsened shortness of breath, sore throat, cough, body or muscle aches and pain, and headache) and illness onset within the prior 7 days. Home visits are scheduled for participants who report a qualifying illness. During each home visit, trained staff collect a respiratory specimen using mid-turbinate nasal and oropharyngeal swabs and ask the participant to describe symptom severity and any disruption to normal activities due to the illness (online supplementary annex 9-acute illness interview). One respiratory specimen was collected within 7 days of illness onset for each qualifying illness. Respiratory specimens collected during home visits are then stored in a cool box immediately, and maintained at $2^{\circ} \mathrm{C}-8^{\circ} \mathrm{C}$ during transportation to the laboratory at Suzhou or Yancheng CDC within 24 hours after collection. Approximately 10 days after illness onset, research staff telephone the participant to ask if the illness has resolved and ask about illness when the participant is most ill (online supplementary annex 10illness follow-up interview). Participants who have not recovered at this time will be telephoned every 3 days (up to four times) or until an illness resolution date is identified. Active surveillance will continue until the end of the study. In a small number of participants, we have piloted the use of a symptom diary (online supplementary annex 11-symptom diary) to describe their illness symptoms for each subsequent day during the illness.

\section{Blood draws at baseline and follow-up interviews}

Blood is collected at enrolment and every 12 months after enrolment, in September-December each year, to permit laboratory confirmation of a history of influenza virus infection between subsequent blood draws by haemagglutination inhibition (HAI) assays (see online supplementary appendix section 8 ). We arranged an additional 
blood draw among all participants in Yancheng in AprilJune 2016, after the winter influenza season, to provide extra information to compare infections in the winter and summer influenza seasons, and we will arrange mid-year blood draws in randomly selected participants in subsequent years of follow-up for the same reason. In each blood draw, we collect up to $10 \mathrm{~mL}$ blood (no less than $5 \mathrm{~mL}$ ) using vacutainer tubes with clot activator. After collection, clotted blood tubes are stored in a cool box immediately and maintained at $2^{\circ} \mathrm{C}-8^{\circ} \mathrm{C}$ while being transferred to the laboratory at the city $\mathrm{CDCs}$ within 24 hours after collection.

\section{Laboratory procedures}

Each combined mid-turbinate and oropharyngeal swab specimen will be divided into three to four aliquots; the first aliquot will be for testing of influenza virus type, subtype/sublineage and absolute quantification, and testing of RSV; the second aliquot will be used for confirmation or further testing; and additional aliquots will be stored for future study uses. rRT-PCR will be completed in a reference laboratory of the local city CDC using US Centers for Disease Control and Prevention (US CDC) primers, probes, reagents and protocols for rRT-PCR testing of influenza virus type, subtype/lineage and RSV; and in-house reagents and protocols provided by the University of Hong Kong for the absolute quantification of influenza virus (see online supplementary appendix section 8). ${ }^{51}$ Study sites are part of the national influenza surveillance network in China and complete proficiency tests for influenza annually provided by the provincial CDC or Chinese National Influenza Centre, which is a WHO Collaborating Centre for Reference and Research on Influenza and an Essential Regulatory Laboratory. Study sites also completed PCR proficiency testing for influenza provided by US CDC, and for RSV provided by Quality Control for Molecular Diagnostics.

Each serum specimen will be divided into three to four aliquots. Paired serum specimens for all participants will be tested for antibody responses to vaccine strains and circulating influenza strains by HAI assays using standard methods. ${ }^{52}$ The sera will be tested in serial doubling dilutions from an initial dilution of 1:10, and sera from the same participants will be tested in parallel. Additional details of the laboratory procedures are provided in online supplementary appendix section 8 .

\section{Hospitalisations and deaths}

When we discover through surveillance that a participant is hospitalised for acute illness, we will use a standardised data abstraction tool (online supplementary annex 7hospital case report) to abstract clinical diagnoses, laboratory testing results, interventions (eg, oxygen support, mechanical ventilation), treatments and duration of hospitalisation from hospital records (see online supplementary appendix section 7). Starting from April 2017, we are also actively identifying hospitalisations in the past month on a monthly basis regardless of whether it is related to an acute illness, and collect information on the admission date, type of medical care, duration and reasons for hospitalisation (online supplementary annex 14monthly hospitalisation surveillance form). For participants who die during the study period, information on the date of death and the primary and secondary causes of death will be documented through medical records, death certificates or from family members willing to share this information, and abstracted using a standardised data abstraction tool (online supplementary annex 12death record and online supplementary appendix section 7). Participant consent has been obtained for access to these records.

If a participant seeks to withdraw from the study at any point of the study, a short questionnaire on time and reasons of withdrawal including hospitalisations (online supplementary annex 13-participant withdrawal form) is administered before he/she exits from the cohort to record reasons for withdrawal, allowing us to assess whether the withdrawal might be related to worsening of health or disability.

\section{Electronic data capture}

All participant encounters including screening for eligibility and follow-up interviews in-person and by telephone calls are being logged, and data immediately captured in an electronic database using REDCap (Research Electronic Data Capture), which is a browser-based, metadata-driven software system (Vanderbilt University, Nashville, Tennessee, USA ${ }^{53}$; the system is also being used to track real-time workflow and other study activities, such as extraction of information from hospitalisation and mortality records, and collection of blood and respiratory specimens (see online supplementary appendix section 9 ). This system permits prespecification of normal ranges and flagging of unusual or missing data for real-time verification (see online supplementary appendix table 13).

\section{FINDINGS TO DATE}

We enrolled 1532 participants into the cohort (table 1). Ninety-eight per cent $(1506 / 1532)$ of the participants finished the enrolment 2 interview, which was the main source of baseline data. Almost all questions were answered. The three questions with the greatest missing value are listed below: $5 \%$ failed to complete the question on self-rated current health on a $0-100$ scale; $2 \%$ did not provide information on their personal annual income; and $1 \%$ did not provide information on their annual household income. We found that 23\% (344/1532) of the participants had ever heard of influenza vaccine at enrolment, and among that subgroup only 4\% (13/344) reported having ever received influenza vaccine, that is, as expected there was very low influenza vaccination coverage $(0.8 \% ; 13 / 1532)$ in our cohort at enrolment.

In the first year of the study (by 3 September 2016), with active surveillance started in late December 2015, we were able to reach the participants and 
confirm their health status in $49412 / 51858$ (95\%) person-weeks of follow-up, equivalent to an average of 32 person-weeks of follow-up per participant (see online supplementary appendix table 11). From 27 December 2015 to 3 September 2016, 186 qualifying acute illnesses were identified in 148 participants in Suzhou, from which 154 (83\%) swabs were collected within 7 days of illness onset. From 11 January 2016 to 3 September 2016, 153 qualifying acute illnesses were identified in 136 participants in Yancheng, from which $146(95 \%)$ swabs were collected.

By the end of the first year of the study, $17(1 \%)$ participants had died, while $47(3 \%)$ participants had withdrawn, including $26 / 47(55 \%)$ who said they had lost interest in participating, 9/47 (19\%) who said they no longer had time to participate, $8 / 47$ (17\%) who were no longer willing to provide samples, $6 / 47$ (13\%) due to severe illness, $5 / 47(11 \%)$ due to family members' objections, $5 / 47(11 \%)$ due to relocation to other cities, $3 / 47(6 \%)$ due to multiple contact failures and $1 / 47(2 \%)$ due to starting warfarin. The sum of reasons for withdrawal exceeds $100 \%$ because some participants reported more than one reason for withdrawal.

\section{FUTURE PLANS}

Currently we are conducting rRT-PCR and HAI assays for influenza, rRT-PCR for RSV and analysing data from illness episodes to identify the incidence and describe the clinical characteristics of influenza virus and RSV infections in older adults. We are also examining data on the knowledge, attitudes and practices related to the influenza virus and vaccines in older adults. We plan to investigate the potential impact of influenza virus and RSV infections on frailty and functional status longitudinally to determine the association of pre-existing immune status with protection against influenza and RSV infection in unvaccinated older adults, and to assess the exposure to avian influenza viruses in this population as $\mathrm{H} 5 \mathrm{~N} 1$ and $\mathrm{H} 7 \mathrm{~N} 9$ infections have been reported in this region.

\section{Strengths and limitations}

This project promises to expand our knowledge of the frequency, clinical features and antibody responses associated with influenza and RSV disease among older adults. These insights could aid China and other countries in making more precise estimates of disease burden and inform decisions about the potential value of existing and new vaccines and other prevention and treatment strategies. The strengths of our cohort include the systematic approach to enrolment with a high enrolment rate, which should permit generalisability of findings to the underlying population, which is older adults residing in rural and urban settings in China. Other strengths include the detailed baseline information, the stored blood and respiratory specimens, active surveillance for illnesses with high compliance, the retention of a high proportion of participants in follow-up, and electronic capture of study data and activities allowing real-time data quality assurance efforts. The weaknesses are the moderate sample size, in the context of relatively low incidence of influenza virus and RSV infections each year, the potential for selection bias in enrolment which would limit generalisability, and the difficulty in maintaining active surveillance and preventing response fatigue and dropouts. The intensity and impact of influenza epidemics can vary from year to year, and antigenic drift in circulating viruses can hinder analyses of immunity using data pooled across years.

\section{Author affiliations}

${ }^{1}$ WHO Collaborating Centre for Infectious Disease Epidemiology and Control, Li Ka Shing Faculty of Medicine, School of Public Health, University of Hong Kong, Hong Kong Special Administrative Region, Hong Kong, China

${ }^{2}$ Chinese National Influenza Center, National Institute for Viral Disease Control and Prevention, Collaboration Innovation Center for Diagnosis and Treatment of Infectious Diseases, Chinese Center for Disease Control and Prevention, Beijing, China

${ }^{3}$ Key Laboratory for Medical Virology, National Health and Family Planning Commission, Beijing, China

${ }^{4}$ Jiangsu Provincial Center for Disease Prevention and Control, Nanjing, China

${ }^{5}$ Suzhou Center for Disease Prevention and Control, Suzhou, China

${ }^{6}$ Yancheng Center for Disease Prevention and Control, Yancheng, China

${ }^{7}$ Centers for Disease Control and Prevention, Atlanta, Georgia, USA

${ }^{8}$ Abt Associates, Cambridge, Massachusetts, USA

${ }^{9}$ School of Public Health, Fudan University, Key Laboratory of Public Health Safety, Ministry of Education, Shanghai, China

${ }^{10}$ Division of Infectious Disease, Key Laboratory of Surveillance and Early-warning on Infectious Disease, Chinese Center for Disease Control and Prevention, Beijing, China

Acknowledgements The authors would like to thank Eduardo Azziz-Baumgartner, Steve Lindstrom and Jerome Tokars for helpful comments and technical support.

Collaborators CARES investigators also include Vicky J Fang, Yi Guan, Chi Kin Lam, Qian Xiong and JS Malik Peiris (the University of Hong Kong); Min Levine and Brett Whitaker (US CDC); William Campbell (Abt Associates); Qigang Dai (Jiangsu CDC); Lin Luan, Benfeng Zheng and Yu Xia (Suzhou CDC); Renjie Jiang, Hongjun Zhang and Guoqing Chen (Yancheng CDC); and Luzhao Feng and Jiandong Zheng (China CDC).

Contributors This project is a multinational collaboration between investigators in the USA, Hong Kong and mainland China, and has required substantial contributions from the coinvestigators. In terms of the specific contributions, the study protocol was designed by BJC and MGT and drafted by CX. Substantial input was provided by FT, JZ, JS, FH, RW, NHLL, CG, ADI, PS, YiS, YuS and HY on the study design in the planning phase in a series of meetings in the USA, Hong Kong and mainland China. The study instruments were prepared by CX and NHLL with input from BJC, FT, JZ, JS, FH, RW, CG, ADI, PS, YiS, RZ, LK, YC, YUS, HY and MGT. The laboratory testing protocols were developed by LK, DKWC and $\mathrm{HZ}$. This cohort profile manuscript was drafted by BJC; the appendix sections were drafted by BJC, FH, RW, NHLL, CG, ADI, $\mathrm{PS}, \mathrm{YiS}, \mathrm{RZ}, \mathrm{LK}, \mathrm{YC}$ and MGT; and all authors provided critical review of the text and approved the final version.

Funding This study was financially supported by the Influenza Division of the US Centers for Disease Control and Prevention (contract no HHSD2002013M53890B: 200-2014-F-60406, 'The epidemiology and prevention of influenza virus infections in low- and middle-income countries'), initially through a research contract with Abt Associates and the University of Hong Kong for the first year, and subsequently through a cooperative agreement with the University of Hong Kong (grant no 1U01IP001064) for the second year. The Suzhou Centers for Disease Control and Prevention (CDC) and Yancheng CDC were contracted to carry out study activities, with the support from China CDC at the national level and Jiangsu CDC at the provincial level. Principal investigators and coinvestigators from US CDC and 
Abt have made substantial contributions to the design of the study protocol and instruments.

Competing interests BJC has received research funding from Sanofi Pasteur. The authors report no other potential conflicts of interest.

Ethics approval The study protocol received ethical approval from the Institutional Review Board of the University of Hong Kong (Ref: UW15 404), and the Ethics Committee of Jiangsu Provincial Center for Disease Prevention and Control (Ref: JSJK2015-B013-02).

Provenance and peer review Not commissioned; externally peer reviewed.

Data sharing statement We intend to make data freely available in the public domain after publication of major findings. Researchers interested in collaborations should contact the principal investigators, BJC (bcowling@hku.hk) and MGT (isq8@ cdc.gov). We are keen to obtain full value from our cohorts and would welcome proposals to use this cohort from potential collaborators or from researchers interested in investigating specific questions independently.

Open Access This is an Open Access article distributed in accordance with the Creative Commons Attribution Non Commercial (CC BY-NC 4.0) license, which permits others to distribute, remix, adapt, build upon this work non-commercially, and license their derivative works on different terms, provided the original work is properly cited and the use is non-commercial. See: http://creativecommons.org/ licenses/by-nc/4.0/

(c) Article author(s) (or their employer(s) unless otherwise stated in the text of the article) 2017. All rights reserved. No commercial use is permitted unless otherwise expressly granted.

\section{REFERENCES}

1. Lozano R, Naghavi M, Foreman K, et al. Global and regional mortality from 235 causes of death for 20 age groups in 1990 and 2010: a systematic analysis for the Global Burden of Disease Study 2010. Lancet 2012;380:2095-128.

2. Falsey AR, Walsh EE. Viral pneumonia in older adults. Clin Infect Dis 2006;42:518-24.

3. Thompson WW, Shay DK, Weintraub E, et al. Influenza-associated hospitalizations in the United States. JAMA 2004;292:1333-40.

4. Centers for Disease Control and Prevention (CDC). Estimates of deaths associated with seasonal influenza-United States, 19762007. MMWR 2010;59:1057-62.

5. Feng L, Shay DK, Jiang $\mathrm{Y}$, et al. Influenza-associated mortality in temperate and subtropical Chinese cities, 2003-2008. Bull World Health Organ 2012;90:279-88.

6. Bloom-Feshbach K, Alonso WJ, Charu V, et al. Latitudinal variations in seasonal activity of influenza and respiratory syncytial virus (RSV): a global comparative review. PLoS One 2013;8:e54445.

7. Borchers AT, Chang C, Gershwin ME, et al. Respiratory syncytial virus-a comprehensive review. Clin Rev Allergy Immunol 2013;45:331-79.

8. Jefferson T, Di Pietrantonj C, Al-Ansary LA, et al. Vaccines for preventing influenza in the elderly. Cochrane Database Syst Rev 2010. CD004876.

9. Beyer WE, McElhaney J, Smith DJ, et al. Cochrane re-arranged: support for policies to vaccinate elderly people against influenza. Vaccine 2013;31:6030-3.

10. Graham BS. Vaccines against respiratory syncytial virus: The time has finally come. Vaccine 2016;34:3535-41.

11. PATH. RSV Vaccine and mAb Snapshot. 2016 http://sites.path.org/ vaccinedevelopment/files/2016/09/RSV-snapshot-September2016. pdf (accessed 8 Nov 2016).

12. World Health Organization. Meeting of the Strategic Advisory Group of Experts on immunization, April 2012-conclusions and recommendations. Wkly Epidemiol Rec 2012:201-16.

13. World Health Organization. Prevention and control of influenza pandemics and annual epidemics. 2003. http://www.who.int/ immunization/sage/1_WHA56_19_Prevention_and_control_of_ influenza_pandemics.pdf (accessed 4 Oct 2016).

14. Palache A, Oriol-Mathieu V, Fino M, et al. Seasonal influenza vaccine dose distribution in 195 countries (2004-2013): Little progress in estimated global vaccination coverage. Vaccine 2015;33:5598-605.

15. Zhou L, Su Q, Xu Z, et al. Seasonal influenza vaccination coverage rate of target groups in selected cities and provinces in China by season (2009/10 to 2011/12). PLoS One 2013;8:e73724.

16. Glezen WP. Morbidity associated with the major respiratory viruses. Pediatr Ann 1990;19:535-42.
17. Fleming DM, Cross KW, Crombie DL, et al. Respiratory illness and mortality in England and Wales. A study of the relationships between weekly data for the incidence of respiratory disease presenting to general practitioners, and registered deaths. Eur J Epidemiol 1993;9:571-6.

18. Chunara R, Goldstein E, Patterson-Lomba O, et al. Estimating influenza attack rates in the United States using a participatory cohort. Sci Rep 2015;5:9540.

19. Rehn M, Carnahan A, Merk H, et al. Evaluation of an Internet-based monitoring system for influenza-like illness in Sweden. PLoS One 2014;9:e96740.

20. Patterson-Lomba O, Van Noort S, Cowling BJ, et al. Utilizing syndromic surveillance data for estimating levels of influenza circulation. Am J Epidemiol 2014;179:1394-401.

21. Hall CE, Cooney MK, Fox JP. The Seattle virus watch. IV. Comparative epidemiologic observations of infections with influenza $A$ and $B$ viruses, 1965-1969, in families with young children. Am J Epidemiol 1973;98:365-80.

22. Monto AS, Koopman JS, Bryan ER. The Tecumseh Study of Illness. XIV. Occurrence of respiratory viruses, 1976-1981. Am J Epidemiol 1986;124:359-67.

23. Petrie JG, Ohmit SE, Cowling BJ, et al. Influenza transmission in a cohort of households with children: 2010-2011. PLoS One 2013;8:e75339.

24. Cowling BJ, Perera RA, Fang VJ, et al. Incidence of influenza virus infections in children in Hong Kong in a 3-year randomized placebocontrolled vaccine study, 2009-2012. Clin Infect Dis 2014;59:517-24

25. Fowler KB, Gupta V, Sullender W, et al. Incidence of symptomatic $\mathrm{A}(\mathrm{H} 1 \mathrm{N1})$ pdm09 influenza during the pandemic and postpandemic periods in a rural Indian community. Int $\mathrm{J}$ Infect Dis 2013;17:e1182-5

26. Hayward AC, Fragaszy EB, Bermingham A, et al. Comparative community burden and severity of seasonal and pandemic influenza: results of the Flu Watch cohort study. Lancet Respir Med 2014;2:445-54.

27. McClure DL, Kieke BA, Sundaram ME, et al. Seasonal incidence of medically attended respiratory syncytial virus infection in a community cohort of adults $\geq 50$ years old. PLoS One 2014;9:e102586.

28. Reed C, Chaves SS, Daily Kirley P, et al. Estimating influenza disease burden from population-based surveillance data in the United States. PLoS One 2015;10:e0118369.

29. Warren-Gash C, Hayward AC, Hemingway H, et al. Influenza infection and risk of acute myocardial infarction in England and Wales: a CALIBER self-controlled case series study. J Infect Dis 2012;206:1652-9.

30. Siriwardena AN, Asghar Z, Coupland CC. Influenza and pneumococcal vaccination and risk of stroke or transient ischaemic attack-matched case control study. Vaccine 2014;32:1354-61.

31. Barker WH, Borisute $\mathrm{H}$, Cox C. A study of the impact of influenza on the functional status of frail older people. Arch Intern Med 1998;158:645-50.

32. Guan XR, Jiang LX, Ma XH, et al. Respiratory syncytial virus infection and risk of acute myocardial infarction. Am J Med Sci 2010;340:356-9.

33. Volling C, Hassan K, Mazzulli T, et al. Respiratory syncytial virus infection-associated hospitalization in adults: a retrospective cohort study. BMC Infect Dis 2014;14:665.

34. Haq K, McElhaney JE. Ageing and respiratory infections: the airway of ageing. Immunol Lett 2014;162:323-8.

35. Fulop T, McElhaney J, Pawelec G, et al. Frailty, Inflammation and Immunosenescence. Interdiscip Top Gerontol Geriatr 2015;41:26-40.

36. $\mathrm{Yu} \mathrm{H}$, Alonso WJ, Feng $\mathrm{L}$, et al. Characterization of regional influenza seasonality patterns in China and implications for vaccination strategies: spatio-temporal modeling of surveillance data. PLoS Med 2013;10:e1001552

37. Borson S, Scanlan J, Brush M, et al. The mini-cog: a cognitive 'vital signs' measure for dementia screening in multi-lingual elderly. Int $J$ Geriatr Psychiatry 2000;15:1021-7.

38. Molloy DW, Alemayehu E, Roberts R. Reliability of a Standardized Mini-Mental State Examination compared with the traditional MiniMental State Examination. Am J Psychiatry 1991;148:206-7.

39. Goldman N, Cornman JC, Chang MC. Measuring subjective social status: a case study of older Taiwanese. J Cross Cult Gerontol 2006;21:71-89.

40. Thompson MG, Gaglani MJ, Naleway A, et al. Changes in self-rated health and subjective social status over time in a cohort of healthcare personnel. J Health Psychol 2014;19:1185-96.

41. Wu C, Gong Y, Wu J, et al. Chinese Version of the EQ-5D Preference Weights: Applicability in a Chinese General Population. PLoS One 2016;11:e0164334 
42. Suurmeijer TP, Doeglas DM, Moum T, et al. The Groningen Activity Restriction Scale for measuring disability: its utility in international comparisons. Am J Public Health 1994;84:1270-3.

43. Gobbens RJ, van Assen MA, Luijkx KG, et al. The Tilburg Frailty Indicator: psychometric properties. J Am Med Dir Assoc 2010;11:344-55.

44. Gobbens RJ, van Assen MA, Schalk MJ. The prediction of disability by self-reported physical frailty components of the Tilburg Frailty Indicator (TFI). Arch Gerontol Geriatr 2014;59:280-7.

45. Bielderman $A$, van der Schans CP, van Lieshout MR, et al. Multidimensional structure of the Groningen Frailty Indicator in community-dwelling older people. BMC Geriatr 2013;13:86

46. Lawton MP, Brody EM. Assessment of older people: selfmaintaining and instrumental activities of daily living. Gerontologist 1969;9:179-86.

47. Campbell VA, Crews JE, Moriarty DG, et al. Surveillance for sensory impairment, activity limitation, and health-related quality of life among older adults-United States, 1993-1997. MMWR CDC Surveill Summ 1999;48:131-56.
48. Centers for Disease Control and Prevention. Behavioral Risk Factor Surveillance System. http://www.cdc.gov/brfss/ (accessed 19 Oct 2016).

49. Searle SD, Mitnitski A, Gahbauer EA, et al. A standard procedure for creating a frailty index. BMC Geriatr 2008;8:24.

50. Chinese National Influenza Center. Chinese Influenza Weekly Report 2016 http://www.chinaivdc.cn/cnic/en/Surveillance/WeeklyReport/ 201606/t20160606_130787.htm (accessed 6 Oct 2016).

51. Lau LL, Cowling BJ, Fang VJ, et al. Viral shedding and clinical illness in naturally acquired influenza virus infections. $J$ Infect Dis 2010;201:1509-16.

52. World Health Organization Global Influenza Surveillance Network. Serological diagnosis of influenza by haemagglutination inhibition testing. Manual for the laboratory diagnosis and virological surveillance of influenza. Geneva, Switzerland: WHO, 2011.

53. Harris PA, Taylor R, Thielke R, et al. Research electronic data capture (REDCap)-a metadata-driven methodology and workflow process for providing translational research informatics support. J Biomed Inform 2009;42:377-81. 